\title{
Análise do efeito de mistura sobre a celeridade do transitório hidráulico em estação elevatória de esgoto via medição indireta e formulações analíticas
}

\section{Analysis of the effect of mixing on the wavespeed of the hydraulic transient in sewage pumping station via indirect measurement and analytical formulations}

\author{
Data de entrada: \\ 04/03/2020 \\ - Data de aprovação: \\ $11 / 08 / 2020$
}

Rodrigo Alexandre da Silva Spakovskis ${ }^{1 *}$ | Edevar Luvizotto Júnior ${ }^{1}$

ORCID ID

Spakovskis RAS (D) https://orcid.org/0000-0002-3695-8726
DOI: https://doi.org/10.36659/dae.2022.005

Luvizotto Júnior E (D) https://orcid.org/0000-0002-4606-4996

\section{Resumo}

Estabelecer um valor adequado para a celeridade é difícil em misturas, como as compostas por líquido, gás e sólido, típicas de sistemas de esgotamento sanitário. $O$ presente estudo propõe uma metodologia que compara os valores da celeridade obtida de forma indireta, por meio de registros de pressões em campo, com os valores obtidos por formulações propostas em literatura para misturas gasosas. Os resultados de campo indicam valores de celeridades diferentes dos obtidos diretamente com os das formulações, fornecendo um indicativo da aderência, ou não, de cada formulação aos resultados empíricos. Usando um algoritmo para retornar o valor da celeridade de cada formulação proposta ao valor da celeridade empírica, é possível observar o comportamento gráfico de cada formulação analisada, bem como estabelecer um percentual típico de volume de gás dissolvido na massa líquida em um sistema de bombeamento de esgotos sanitários.

Palavras-chave: Transitório hidráulico. Celeridade. Estação elevatória de esgoto.

\section{Abstract}

Establishing an adequate value for wave speed is very difficult in mixtures, such as those composed of liquid, gas and solid, typical of sanitary sewage systems. The present study proposes a methodology that compares the values of the wave speed obtained indirectly, by means of pressure records in the field, with the values obtained by formulations proposed in the literature for gas mixtures. The field results indicate wave speed values different from those obtained directly with those of the formulations, providing an indication of the adherence or not of each formulation to the empirical results. Using an algorithm to return the value of the wave speed of each proposed formulation to the value of the empirical wave speed, it is possible to observe the graphic behavior of each formulation analyzed as well as establish a typical percentage of volume of gas dissolved in the liquid mass in a sewage pumping system Sanitary.

Keywords: Hydraulic transient. Wave speed. Sewage pumping station.

\footnotetext{
${ }^{1}$ Unicamp - Universidade Estadual de Campinas - Campinas - São Paulo - Brasil.

* Autor correspondente: rspakovskisasabesp.com.br.
} 


\section{INTRODUÇÃO}

As operações das Estações Elevatórias de Esgoto (EEEs) envolvem a acumulação dos efluentes em poços, recalque do esgoto e esvaziamento do poço de acumulação. Esse processo de operação é cíclico, e os parâmetros hidráulicos como pressão e vazão são determinados para as condições de regime permanente, ou seja, não são consideradas alterações significativas dos valores desses parâmetros.

A condição denominada transitória decorre de variações bruscas dos parâmetros de interesse e que possam ocorrer durante o regime permanente inicial, como partida ou parada de conjunto motobomba, por exemplo. A celeridade é uma das grandezas que governam o fenômeno transitório, definindo sua intensidade e frequência, e sua valoração é fundamental para a descrição e a quantificação do fenômeno.

O esgoto, apesar de ter uma composição predominante de fase líquida, apresenta percentuais de fase sólida e gasosa que interferem em variáveis responsáveis pela resposta transitória, como a celeridade. Segundo Wylie e Streeter (1978), a celeridade de um líquido com uma pequena quantidade de gases dissolvidos é consideravelmente menor do que no líquido puro. Os pesquisadores citam que os experimentos realizados por Kobori et al. (1955) e Pearsall (1965), em estações de bombeamento de esgotos, mostraram que a redução na celeridade é de cerca de $75 \%$ em relação a um líquido puro, como a água, dependendo do teor de gás.

O presente trabalho tem como objetivos determinar quais equações analisadas, propostas em literatura, representam tipicamente a instalação estudada, e estabelecer um percentual típico de gás dissolvido na massa líquida nessa mesma instalação.

Para alcançar esses objetivos, foi desenvolvida uma metodologia que buscou, por meio de um algoritmo usado em software comercial, comparar os valores empírico e teórico da celeridade, metodologia esta não observada em qualquer trabalho pesquisado. A observação gráfica, resultante do processo de otimização do algoritmo, possibilita a obtenção das equações que se ajustam à instalação, em comparação com um "gráfico referência", e a solução apresentada pelo algoritmo possibilita o estabelecimento de um valor médio percentual de gás dissolvido para a instalação estudada.

\section{FUNDAMENTAÇÃO TEÓRICA - REVISÃO BIBLIOGRÁFICA}

Para Boulos et al. (2005), o transitório hidráulico pode ser entendido como a conversão da energia cinética transportada pelo líquido em energia de tensão nas paredes do tubo, causando uma "onda de pulso" de pressão anormal que irá perturbar a instalação. $O$ som de martelar que as vezes é ouvido indica que uma parte da energia cinética do líquido original é convertida não só em pressão, mas também em energia sonora. A Fig. 1 ilustra a variação de pressão no transcorrer de um determinado tempo $(\Delta t)$ após o fechamento de uma válvula à jusante da seção de análise. Na figura é possível observar os regimes permanente inicial, final e o regime transitório que transcorre num intervalo TRT, onde ocorre a adequação entre os dois regimes permanentes. 


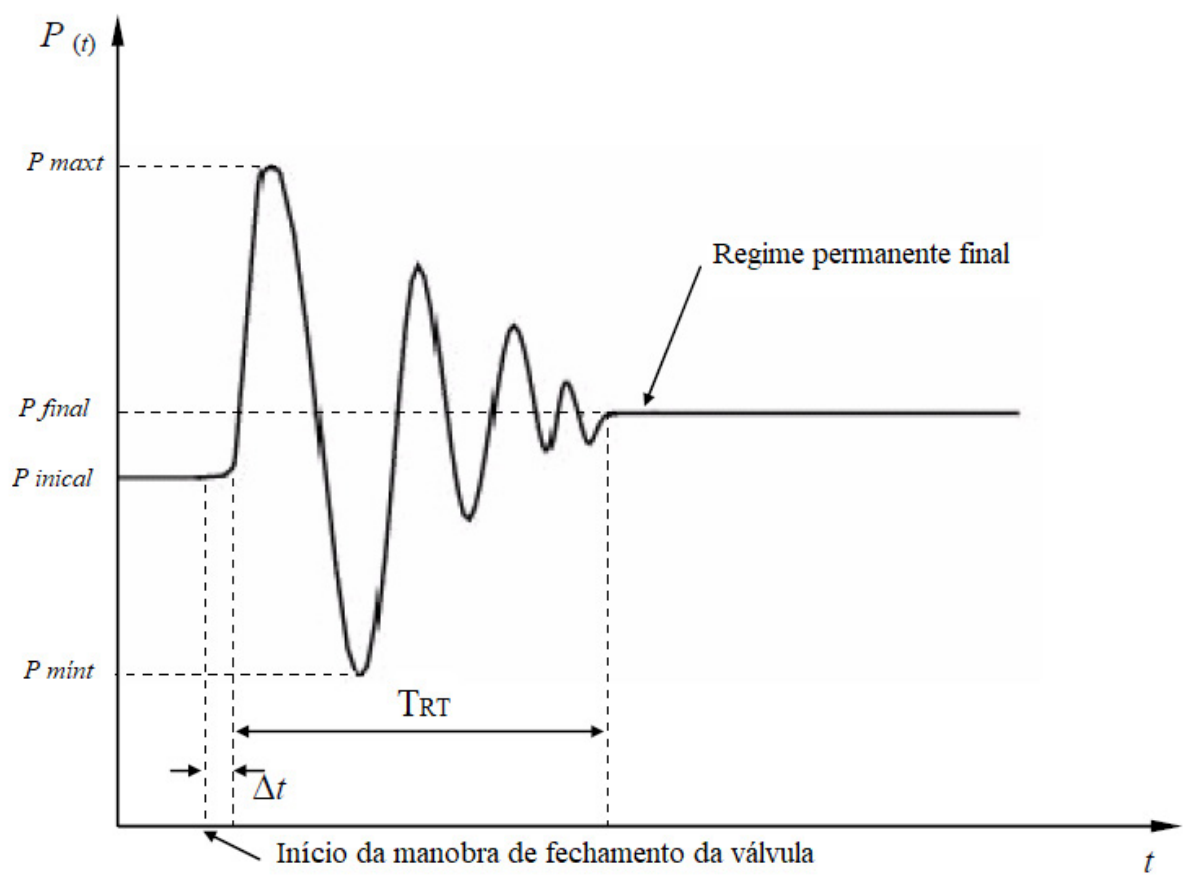

Figura 1 - Escoamentos em regime permanente e transitório hidráulico Fonte: Adaptado de Elbashir e Amoah (2007)

O cientista russo Nikolai Egorovich Joukowsky (1897) (LÜDECKE et al. (2006) e GUIDAOUI et al. (2005)), propôs uma equação simples para avaliar a variação de pressão $(\Delta p)$ como função da variação instantânea da velocidade $(\Delta \mathrm{V})$. Essa equação é conhecida como equação de Joukowsky (Eq. 1).

$\Delta p=+/-$ p.a. $\Delta V$

Ou, em termos de carga de pressão,

$\Delta \mathrm{H}=+/-\mathrm{a} / \mathrm{g} \cdot \Delta \mathrm{V}$

Sendo, $\Delta p$ = variação da pressão na seção de controle $(\mathrm{m}) ; \rho=$ massa específica do fluido $\left(\mathrm{kg} / \mathrm{m}^{3}\right)$; $\mathrm{a}=$ celeridade $(\mathrm{m} / \mathrm{s}) ; \Delta \mathrm{V}=$ variação da velocidade média na seção de controle $(\mathrm{m} / \mathrm{s})$.

A compressão e a descompressão da tubulação, expressas pela mudança de sinal em (1), ocorrem de maneira cíclica, e o período " $T$ " das oscilações das cargas de pressão da tubulação pode ser descrito de forma ilustrativa como na Fig. 2, para uma instalação hipotética, com atrito negligenciado, composta por um reservatório a montante e a um nível constante, uma tubulação e uma válvula a jusante com manobra de fechamento instantâneo. A Fig. 2 ilustra o ciclo de compressão e descompressão na seção da válvula após o fechamento brusco, mostrando que esse ciclo tem um período bem definido $(T=4 L / a)$, que depende exclusivamente do comprimento da tubulação e da celeridade da onda de pressão (a). 


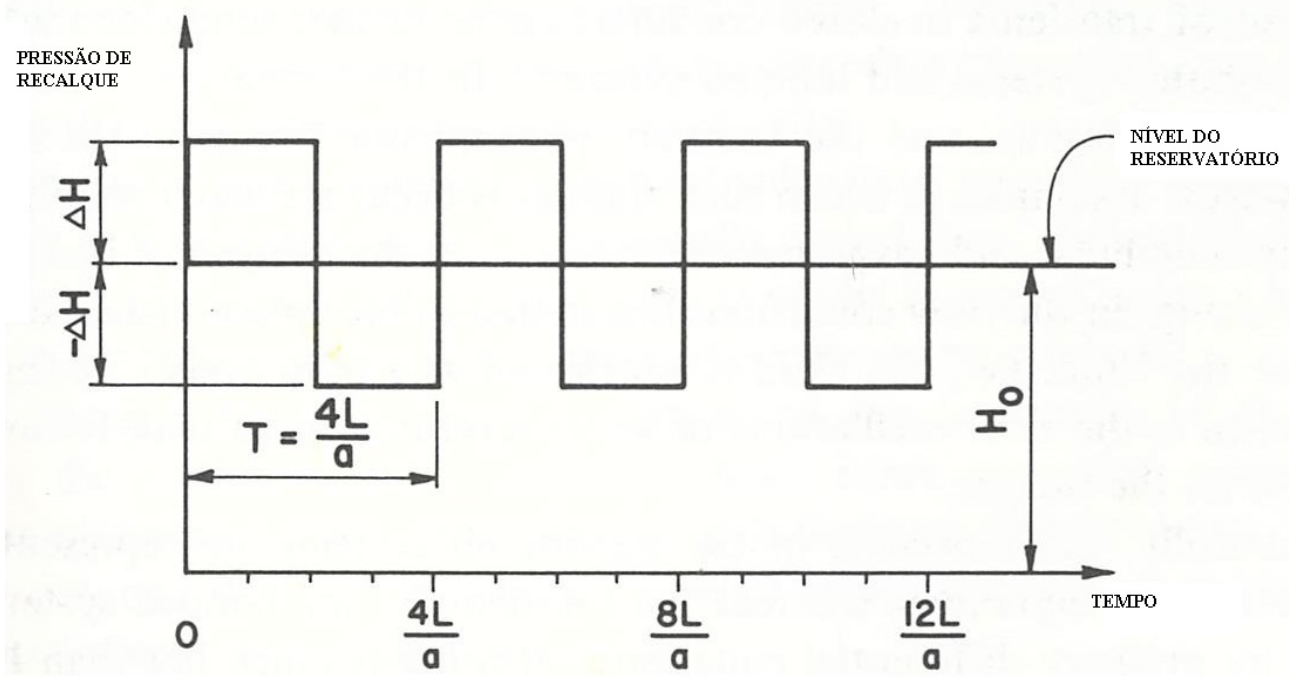

Figura 2 - Período de uma instalação Fonte: Adaptado de Chaudry (1979)

Assim, se esse período pode ser obtido pela interpretação do registro de sinal de pressão na seção, a celeridade pode ser avaliada por meio da Eq. 3,

$$
\boldsymbol{a}=\frac{4 L}{T}
$$

Sendo, $T$ = período do fenômeno físico (s); $L=$ comprimento total da tubulação $(\mathrm{m})$ e $a=$ celeridade $(\mathrm{m} / \mathrm{s})$.

O comportamento ilustrado na Fig. 2 reflete, para a situação descrita, o que se obtém como solução das equações que governam o fenômeno transitório, obtidas originalmente por Allievi (1903), com base nas equações de conservação de massa (4) e de quantidade de movimento (5) considerando o atrito:

$$
\frac{a^{2}}{g A} \frac{\partial Q}{\partial x}+\frac{\partial H}{\partial t}=0
$$

$$
\frac{\partial Q}{\partial t}+g A \frac{\partial H}{\partial x}+\frac{f}{2 D A} Q|Q|=0
$$

Sendo, $Q$ = vazão $\left(\mathrm{m}^{3} / \mathrm{s}\right) ; H=$ carga piezométrica $(\mathrm{m})$; $g=$ aceleração da gravidade $\left(\mathrm{m} / \mathrm{s}^{2}\right) ; f=$ fator de atrito da fórmula universal da perda de carga (em regime permanente); $D$ = diâmetro da tubulação (m); $A$ = área da seção transversal da tubulação $\left(\mathrm{m}^{2}\right) ; a=$ celeridade $(\mathrm{m} / \mathrm{s}) ; t=$ tempo transcorrido $(\mathrm{s}) ; x=$ distância medida ao longo do eixo da tubulação (m).

No caso ilustrado na Fig. 2, o terceiro termo da equação (5), referente ao atrito, foi negligenciado. Para casos com atrito, embora o amortecimento das ondas ocorra, desfazendo a forma de onda retangular, o comportamento cíclico e periódico é evidente, e o período do fenômeno físico segue a mesma equação apresentada.

As Eq. (4 e5) definem um sistema de equações diferenciais do tipo hiperbólicas usualmente resolvido de forma numérica. Na solução dessas equações a celeridade tem fundamental importância para a quantificação das grandezas de interesse, ou seja, a carga (H) e a vazão (Q), nas seções de análise no transcorrer do tempo. 0 matemático Holandês Diederik Korteweg em 1878, (Abreu et al. (1995) e Chaudry (1979)) desenvolveu a equação para o cálculo da celeridade, que é a propagação de uma onda de pressão ao longo de uma tubulação, em um evento transitório. A Eq. 6 re- 
sulta das considerações adotadas por Korteweg, considerando uma única fase e a tubulação de parede fina com deformação elástica.

$a=\sqrt{\frac{K / \rho}{1+\left(\frac{K}{E}\right)\left(\frac{D}{e}\right) \psi}}$

Sendo, $a=$ celeridade $(\mathrm{m} / \mathrm{s}) ; K=$ módulo de compressibilidade volumétrica do fluido $\left(\mathrm{N} / \mathrm{m}^{2}\right) ; \rho=$ massa específica do fluido $\left(\mathrm{N} / \mathrm{m}^{3}\right) ; E=$ módulo de Young ou módulo de elasticidade do material da tubulação (N/ $\left.\mathrm{m}^{2}\right) ; D=$ diâmetro da tubulação $(\mathrm{m}) ; e=$ espessura das paredes da tubulação $(\mathrm{m}) ; \psi=$ coeficiente relacionado ao tipo de vinculação da tubulação (adimensional).

Segundo Malekpour et al. (2015), a diferença significativa na compressibilidade do ar em relação à da água permite que $\mathrm{o}$ ar possa ser usado para absorver e atenuar pressões transitórias. $\mathrm{O}$ ar ou a mistura gasosa presente no sistema de esgoto sanitário pode alterar a celeridade e modificar as pressões a que este está exposto o que se evidencia na Eq. 1.

Pothof et al. (2011) elencam algumas formas de incorporação de ar nos sistemas elevatórios de esgotos:

- Vazamentos nas redes;
- Jato em queda livre no poço de sucção;

- Processos bioquímicos.

Jönsson (1985) apud Burrows e Qui (1995) afirma que há um limite sobre a quantidade de ar que conduz a amortecimento do transitório, pela sua influência direta sobre a celeridade. Os pesquisadores observaram que enquanto uma grande cavidade de ar atua como um acumulador e suprime o máximo de pressão transitória, após o desligamento de uma bomba por exemplo, esse volume dividido entre várias bolsas de ar pode substancialmente potencializar pressões transitórias. No experimento realizado por Lubbers e Clemens (2005), foram observadas as condições de perfil topológico para atendimento das condições de formação de bolsas da ar. O perfil que atende essas condições tem forma totalmente ascendente, sem grandes deflexões verticais.

A influência que uma pequena quantidade de gases dissolvidos exerce sobre a celeridade é ilustrada na Fig. 3, a qual trataremos também como "gráfico referência". Para Kobori et al. (1955) apud Wylie e Streeter (1978), a presença de gases em sistemas de esgotamento sanitário varia entre 0,1 e $1 \%$ em relação ao volume total.

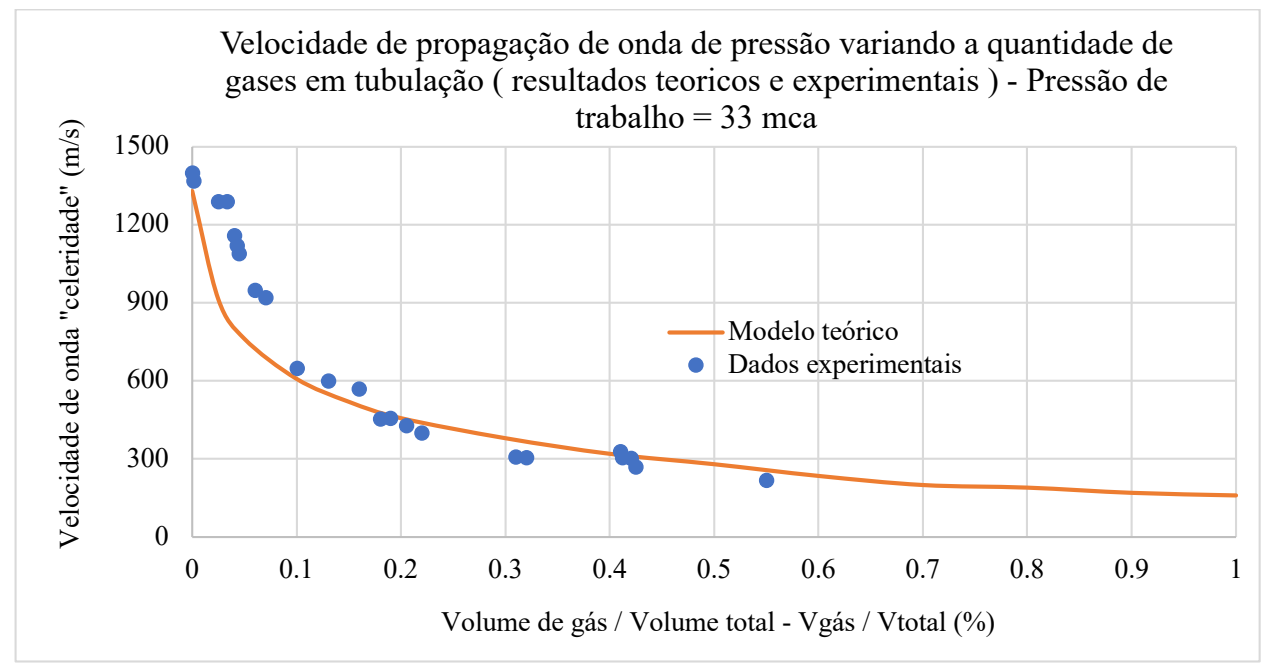

Figura 3 - Celeridade x volume de mistura gás-líquido Fonte: Adaptado de Wylie e Streeter (1978) 
A influência do ar ou gás dissolvido na equação da celeridade não é trivial, pois envolve um processo termodinâmico complexo. Assim, as equações encontradas na literatura baseiam-se em hipóteses simplificadoras, tais como:

- A mistura gás-líquido é homogênea, isto é, as bolhas de gás são distribuídas uniformemente no líquido;

- As bolhas de gás seguem a lei isotérmica;
- A pressão dentro das bolhas é independente da tensão superficial e da pressão de vapor e seu valor não se altera.

Como base em hipóteses dessa natureza, alguns autores propõem equações para a celeridade com incorporação de gases. As Eq. 7, 8, 910 são, respectivamente, apresentadas por Wylie e Streeter (1978), Chaudry (1979), Ewing (1980) e Lee e Pejovic (1996):

$$
a_{W S}=\sqrt{\frac{[K m] /(\rho m)}{\left.1+\llbracket K m \cdot\left(\frac{D}{E e}\right) \cdot \psi\right]}}
$$

$$
\begin{aligned}
& a_{C H}=\sqrt{\left[\left\{\rho l \cdot\left(1-\left[\frac{(\alpha) \cdot P \operatorname{trans}}{P t}\right]\right)\right\}+\rho g \cdot P \operatorname{trans}\right] \cdot\left[\left(\frac{(\alpha) \cdot P \operatorname{Ptrans}}{P t^{2}}\right)+\frac{1}{K l}+\frac{D}{E e}\right] \cdot \psi} \\
& a_{E W}=\left[\left|\left\{(1-\alpha)\left(\frac{\rho m}{K m}\right)\right\}+\left\{\frac{\rho m \cdot \alpha}{P t}\right\}\right|\right]^{-1 / 2} \\
& a_{L P}=\left[\rho l(1-\alpha) \cdot\left(\frac{1}{K l}+\frac{\alpha}{P t}+\frac{\psi \cdot D}{e \cdot E}\right)\right]^{-1 / 2}
\end{aligned}
$$

$\psi=$ tipo de vinculação, ancoragem da tubulação (adimensional); $\alpha$ = relação entre volume do gás e volume total (adimensional).

Sendo,

$$
\begin{aligned}
& K m=\left[\frac{K l}{1+[(\text { Vgas } / V t) \cdot\{(\text { Kl/Kgas })-1\}]}\right] \\
& \rho m=\left(\left[\rho g a s \cdot\left(\frac{V g a s}{V t}\right)\right]+\left[\rho l \cdot\left(\frac{V l}{V t}\right)\right]\right) \\
& \alpha=\frac{V g a s}{V t}
\end{aligned}
$$




\section{METODOLOGIA}

A pesquisa avaliou uma linha de recalque de esgotos com características de perfil suave ascendente, com o intuito da não formação de bolsões de ar que ocupassem a seção total da tubulação, descaracterizando o problema abordado por fenômenos anteriormente descritos por Lubbers e Clemens (2005).

A metodologia empregada considerou o registro de dados de pressão coletados em campo durante a ocorrência de um evento transitório, desligamento de conjunto motobomba, para obtenção indireta do valor da celeridade da instalação, conforme descrito anteriormente na Eq. 3 e, com o valor obtido, investigou-se a aderência das equações propostas por Wylie e Streeter (1978), Chaudry (1979), Ewing (1980) e Lee e Pejovic (1996) ao valor obtido e a quantidade de gás dissolvido necessário para obtê-lo. As incertezas sobre cada uma das variáveis que entram no cômputo das equações analisadas foram limitadas pelas restrições impostas por faixas de valores obtidos da literatura e catálogo de fabricantes.
Foi usado o módulo de otimização baseado no algoritmo Gradiente Reduzido Generalizado (GRG) disponível no solver do Microsoft Excel®, cuja função objetivo é não linear, com restrições lineares. Para Köksoy e Yalcinoz (2006), o GRG é o método muito empregado na resolução de problemas de otimização de múltiplas variáveis, caso das equações analisadas pelo presente estudo, por ser de fácil implantação. A maior dificuldade na utilização do GRG deve-se ao fato de que é necessário que a estimativa inicial das variáveis esteja próxima do valor do ponto ótimo para uma garantia de convergência. Segundo Motulsky e Ransnas (1987), as estimativas iniciais das variáveis podem ou devem ser especificadas tendo em consideração a experiência do investigador ou eventuais análises preliminares. A escolha dos valores iniciais é mais influente e, por isso, mais importante nos casos em que os modelos incluem muitos parâmetros.

No problema analisado envolvendo as equações estudadas, a variável de maior importância é o volume de gás (Vgás) incorporado à mistura. Para uma melhor compreensão do procedimento empregado, apresenta-se o fluxograma da Fig. 4.

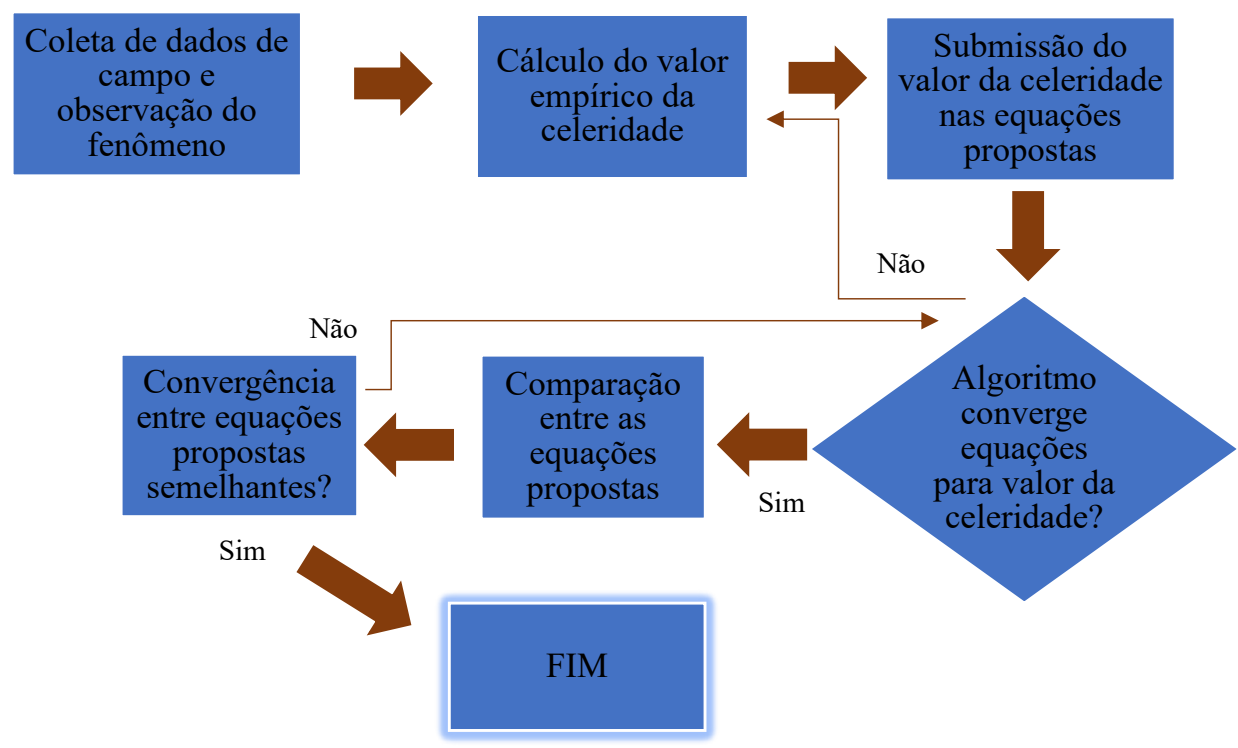

Figura 4 - Fluxograma geral do processo do estudo. 


\section{ESTUDO DE CASO}

O estudo foi realizado no município de Itapecerica da Serra, localizado na Região Metropolitana de São Paulo (RMSP). A linha de recalque de esgotos pertence à EEE Jardim Jacira e possui 1.873,00 metros de extensão; o material da rede é de ferro fundido classe $\mathrm{K} 7$ e a espessura das paredes é de $6,0 \mathrm{~mm}$. A linha de recalque parte da cota $734,00 \mathrm{~m}$ e segue uma linha de aclive suave até a conta $750,00 \mathrm{~m}$, tendo percorrido aproximadamente $800,00 \mathrm{~m}$ de extensão. A partir desse ponto, a inclinação da linha passa a ser mais íngreme até o ponto de descarte, na cota
$801,00 \mathrm{~m}$. No ponto final da linha de recalque, o efluente é lançado em um Poço de Visita (PV) de transição e posteriormente para outra bacia de esgotamento subsequente. A Fig. 5 ilustra o perfil da linha de recalque da EEE Jardim Jacira, tendo como uma de suas principais características o perfil suave e totalmente ascendente.

A bacia de esgotamento da elevatória em estudo possui como característica a contribuição de esgotos basicamente toda do tipo residencial. $\mathrm{Na}$ bacia não há indústria, assim caracterizando os esgotos afluentes na estação como do tipo predominantemente residencial.

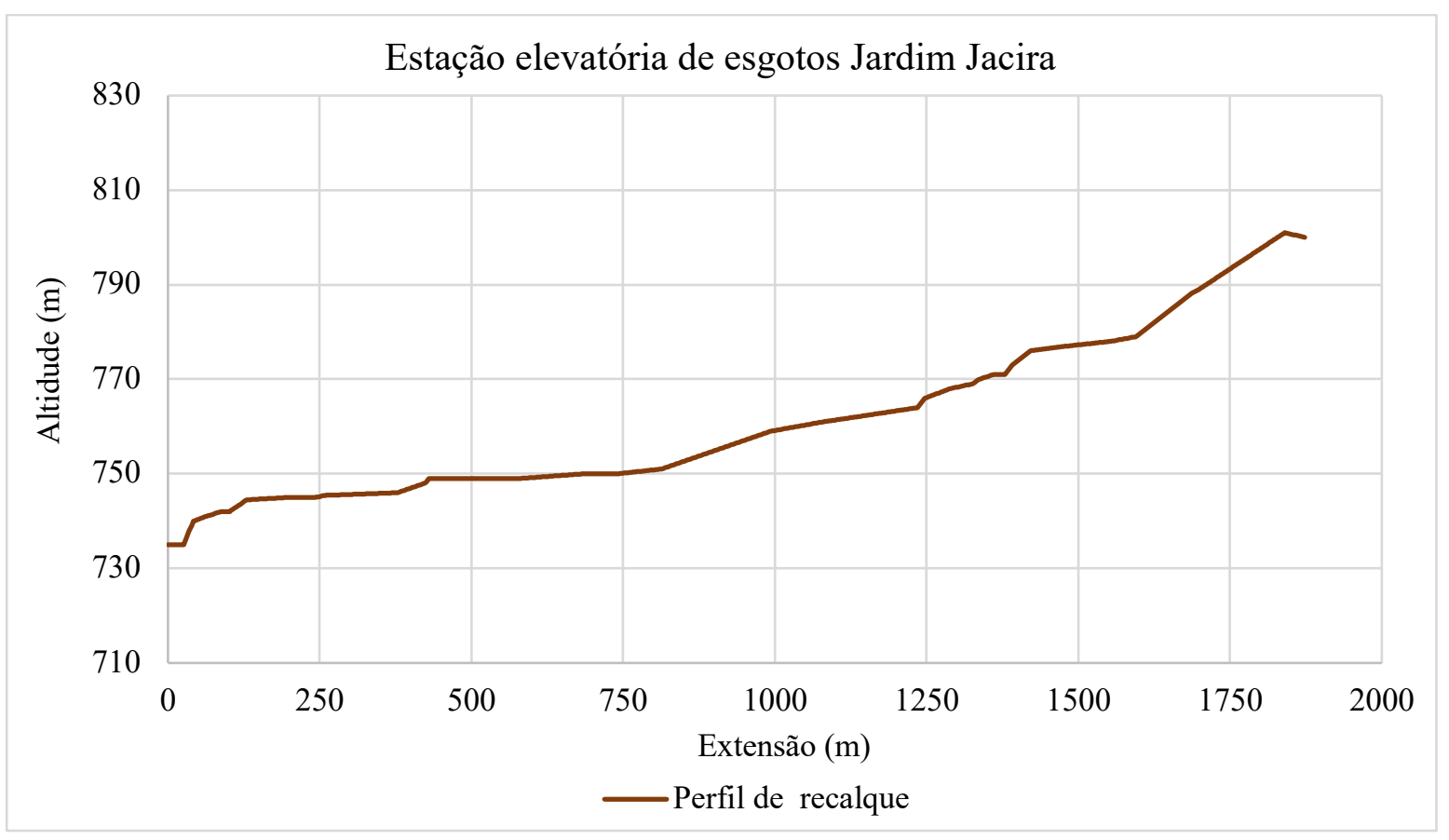

Figura 5 - Perfil da linha de realque da EEE Jardim Jacira.

Foram instalados equipamentos registradores de pressão do tipo "DataLoggers" no barrilete de recalque, após a válvula de retenção. Os equipamentos registravam os dados de pressão a cada
0,5 segundo, e a coleta de dados foi feita no dia 13/09/2019. Foram coletados 360 dados de pressão, e a Fig. 6 apresenta os dados coletados que evidenciam o fenômeno transitório estudado. 


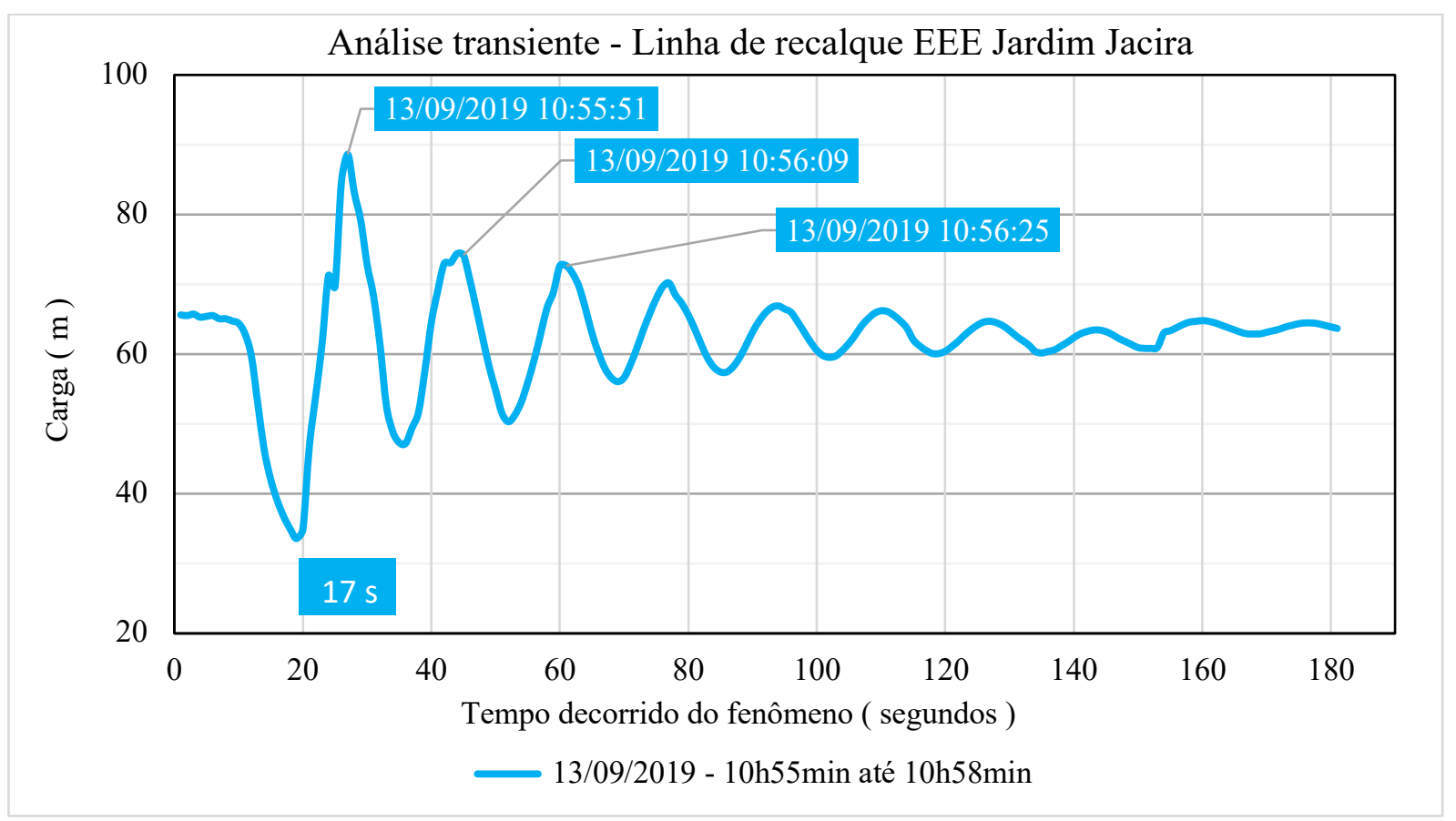

Figura 6 - Coleta de dados das pressões transitórias na EEE Jardim Jacira - Setembro/2019. Fonte: Sabesp (2019)

Observando os dados de campo ilustrados na Fig. 6 pode-se observar que o período "T" do fenômeno é de aproximadamente 17 segundos, e pela Fig. 5 é possível observar que a extensão de toda a rede de recalque é de 1.873,00 metros. Assim é possível obter:

$$
a=\frac{4 \times 1873}{17}=\mathbf{4 4 0 , 7 1} \mathrm{m} / \mathrm{s}
$$

O presente estudo buscou desenvolver a otimização do processo de comparação entre a celeridade de campo, calculada conforme acima, e a celeridade teórica apontada na literatura conforme a revisão bibliográfica apresentada. A função objetivo desenvolvida buscou minimizar a diferença dos valores das celeridades partindo de um valor pré-determinado.

A função objetivo fica:

$$
F(x)=\min \left(a_{\text {campo }}-a_{\text {teórico }}\right)^{2}
$$

Sendo $a_{\text {teórico }}$ o valor aplicado a cada uma das equações propostas na literatura para, $a_{w s}, a_{C H}$, $a_{E W}$ e $a_{L P}$

a) Para a celeridade $a_{w s}$ temos as variáveis em função de:

$g 1(x)=(K m, \rho m, D, E, e, \psi)$

resultando a Eq. 15 na forma:

$F(x)=\min \left(a_{\text {campo }}-a_{g 1(x)}\right)^{2}$

b) Para a celeridade $a_{C H}$ temos as variáveis em função de:

$g 2(x)=\left(\alpha, P_{\text {maxt }}, P_{\text {mint }}, \rho_{\text {gas }}, \rho_{l}, P_{t}, K_{l}, D, E, e, \psi\right)$

resultando a Eq. 16 na forma:

$$
F(x)=\min \left(a_{\text {campo }}-a_{g 2(x)}\right)^{2}
$$


c) Para a celeridade $a_{E W}$ temos as variáveis em função de:

$g 3(x)=\left(K_{m}, \rho_{m}, V_{g}, P_{t}, D, E, e, \psi\right)$

resultando aEq. 17 na forma:

$F(x)=\min \left(a_{\text {campo }}-a_{g 3(x)}\right)^{2}$

d) Para a celeridade a_LP temos as variáveis em função de:

$g 4(x)=\left(\rho_{1}, V_{g}, P_{t}, K_{l}, D, E, e, \psi\right)$

resultando a Eq. 18 na forma:

$$
F(x)=\min \left(a_{\text {campo }}-a_{g 4(x)}\right)^{2}
$$

As Eq. 15, 16, 17 e 18 então foram utilizadas no Solver do Microsoft Excel ${ }^{\circledR}$. As variáveis ( $x$ ) das funções $g_{1 . . . n}(x)$ são os parâmetros que cada equação analisada leva em conta para o tipo de mistura gás-líquido.

Uma primeira estimativa para o presente estudo foi de um volume de gás, cerca de $0,5 \%$, em relação ao volume total. Os valores extremos das variáveis das equações obtidos na literatura foram tomados como restrições do processo de otimização conforme a Tabela 1.

Tabela 1 - Faixa de variação das componentes das equações estudadas

\begin{tabular}{|c|c|c|}
\hline Variável & Faixa dos valores obtidos na literatura & Referência \\
\hline Kl - módulo de elasticidade do líquido $\left(\mathrm{N} / \mathrm{m}^{2}\right)$ & $1,92 \times 10^{9}$ a $2,27 \times 10^{9}$ & $(1)$ e (2) \\
\hline E- módulo de elasticidade do tubo $\left(\mathrm{N} / \mathrm{m}^{2}\right)$ & $0,80 \times 10^{11}$ a $1,70 \times 10^{11}$ & $(1)$ e (2) \\
\hline$\rho l$ - massa específica do líquido $\left(\mathrm{kg} / \mathrm{m}^{3}\right)$ & $9,80 \times 10^{2}$ a $1,05 \times 10^{3}$ & (2) \\
\hline$D$ - Diâmetro do tubo $(\mathrm{m})$ & $3,95 \times 10^{-1}$ a $4,16 \times 10^{-1}$ & $(4)$ \\
\hline$e$ - Espessura das paredes de tubo (m) & $5,95 \times 10^{-3}$ a $6,5 \times 10^{-3}$ & (4) \\
\hline$\psi$ - tipo de vinculação, ancoragem da tubulação (adimensional) & 0,85 a 1 & $(2)$ \\
\hline $\rho g a s$ - massa específica do gás na mistura $\left(\mathrm{N} / \mathrm{m}^{3}\right)$ & $1,23 \times 10^{3}$ a $1,25 \times 10^{3}$ & (3) \\
\hline$\alpha$ - relação Vgás / Vtotal da mistura (\%) & $1,0 \times 10^{-2}$ a 1,0 & (5) \\
\hline$P t$ - pressão de recalque $\left(\mathrm{N} / \mathrm{m}^{2}\right)$ & $4,54 \times 10^{5}$ a $6,85 \times 10^{5}$ & (6) \\
\hline Ptrans - pressão transiente $\left(\mathrm{N} / \mathrm{m}^{2}\right)$ & $2,94 \times 10^{5}$ a $7,85 \times 10^{5}$ & (6) \\
\hline$V_{G}-$ Volume de gás na mistura $\left(\mathrm{m}^{3}\right)$ & $5,0 \times 10^{-2}$ a 1,0 & (5) \\
\hline $\mathrm{K}_{\mathrm{GAS}}=$ módulo de elasticidade do gás $\left(\mathrm{N} / \mathrm{m}^{2}\right)$ & $1,92 \times 10^{5}$ a $2,27 \times 10^{5}$ & (3) \\
\hline
\end{tabular}

(1) PARMAKIAN (1955), (2) CHAUDRY (1979), ${ }^{(3)}$ ABREU et al. (1995), ${ }^{(4)}$ CATÁLOGO SAINT GOBAIN, ${ }^{(5)}$ WILYE e STREETER (1978).

\section{RESULTADOS}

Os resultados obtidos do processo de otimização são apresentados na Tabela 2, que mostra os valores iniciais estimados para a celeridade das equações estudadas, evidenciando a incorreção do percentual de gás dissolvido na estimativa inicial em relação ao valor obtido pela otimização. Também é possível observar as diferenças percentuais de cada variável, evidenciando quais tiveram maior impacto sobre o valor ótimo.
Os valores em negrito destacam os valores dos parâmetros de cada equação analisada que tiveram as maiores alterações verificadas.

Para a análise de resultados será usado como referência o comportamento da celeridade, variando seu percentual de gás assim como na Fig. 3, procurando observar qual das equações analisadas apresentou comportamento semelhante ao do chamado "gráfico referência". 
Tabela 2 - Resultado da otimização das equações propostas

\begin{tabular}{|c|c|c|c|c|c|c|c|c|c|c|c|}
\hline \multicolumn{4}{|c|}{ EQUAÇÕES UTILIZADAS } & \multicolumn{2}{|c|}{$\begin{array}{l}\text { WYLIE e STREETER } \\
\text { (1978) }\end{array}$} & \multicolumn{2}{|c|}{ CHAUDRY (1979) } & \multicolumn{2}{|c|}{ EWING (1980) } & \multicolumn{2}{|c|}{ LEE e PEJOVIC (1996) } \\
\hline \multicolumn{4}{|c|}{ FUNÇÃO OBJETIVO } & 440,71 & \multirow{2}{*}{$-56 \% \sim$} & 440,71 & \multirow{2}{*}{$-17 \% \sim$} & 440,71 & \multirow{2}{*}{$-61 \% \sim$} & 440,71 & \multirow{2}{*}{$-65 \% \sim$} \\
\hline \multicolumn{4}{|c|}{ VALOR INICIAL } & 196,01 & & 364,49 & & 171,24 & & 155,40 & \\
\hline \multicolumn{4}{|c|}{ Diferença em módulo } & \multicolumn{2}{|c|}{244,70} & \multicolumn{2}{|c|}{76,22} & \multicolumn{2}{|c|}{267,47} & \multicolumn{2}{|c|}{285,31} \\
\hline \multicolumn{4}{|c|}{$F(x)=\min \left(a_{\text {campo }}-a_{g 1 \ldots n(x)}\right)^{2}$} & \multicolumn{2}{|c|}{0} & \multicolumn{2}{|c|}{0} & \multicolumn{2}{|c|}{0} & \multicolumn{2}{|c|}{0} \\
\hline \multicolumn{4}{|c|}{ VALOR DA CELERIDADE NO MODELO } & \multicolumn{8}{|c|}{440,71} \\
\hline \multirow{2}{*}{ VARIÁVEIS } & \multicolumn{2}{|c|}{ RESTRIÇÕES } & \multirow{2}{*}{ BASE } & \multirow{2}{*}{$\mathbf{R}^{*}$} & \multirow{2}{*}{ Var.**** } & \multirow{2}{*}{$\mathbf{R}^{*}$} & \multirow{2}{*}{ Var.:** } & & & & \\
\hline & Menor & Maior & & & & & & $\mathbf{R}^{n}$ & $\operatorname{Var}^{*+\ldots}$ & $\mathbf{R}^{*}$ & Var." \\
\hline$K l\left(\mathrm{~N} / \mathrm{m}^{2}\right)$ & $1,92 E+09$ & $2,27 E+09$ & $1,92 E+09$ & $1,92 E+09$ & 0,0 & $1,92 E+09$ & 0,0 & $1,92 E+09$ & 0,0 & $1,92 E+09$ & 0,0 \\
\hline$K_{\text {gas }}\left(\mathrm{N} / \mathrm{m}^{2}\right)$ & $1,92 E+05$ & $2,27 E+05$ & $1,92 E+05$ & $1,92 E+05$ & 0,0 & & & $1,92 E+05$ & 0,0 & & \\
\hline$\rho l\left(\mathrm{~N} / \mathrm{m}^{3}\right)$ & $9,80 \mathrm{E}+02$ & $1,05 E+03$ & $9,80 E+02$ & $1,05 E+03$ & 7,1 & $9,80 \mathrm{E}+02$ & 0,0 & $1,05 E+03$ & 7,1 & $9,80 \mathrm{E}+02$ & 0,0 \\
\hline$E\left(\mathrm{~N} / \mathrm{m}^{2}\right)$ & $8,00 E+10$ & $1,2 \mathrm{E}+11$ & $8,00 E+10$ & $8,00 E+10$ & 0,0 & $8,00 E+10$ & 0,0 & $8,00 E+10$ & 0,0 & $8,00 E+10$ & 0,0 \\
\hline$D(\mathrm{~m})$ & $3,95 \mathrm{E}-01$ & $4,16 \mathrm{E}-01$ & $4,00 \mathrm{E}-01$ & $4,16 \mathrm{E}-01$ & 4,0 & $3,95 \mathrm{E}-01$ & $-1,3$ & $4,00 \mathrm{E}-01$ & 0,0 & $3,95 \mathrm{E}-01$ & $-1,3$ \\
\hline$e(\mathrm{~m})$ & $5,95 \mathrm{E}-03$ & $6,30 \mathrm{E}-03$ & $6,00 \mathrm{E}-03$ & $5,95 \mathrm{E}-03$ & $-0,8$ & $6,08 \mathrm{E}-03$ & 1,3 & $6,00 \mathrm{E}-03$ & 0,0 & $6,30 \mathrm{E}-03$ & 5,0 \\
\hline$\psi$ (adim.) & $8,50 \mathrm{E}-01$ & $1,00 E+00$ & $8,50 \mathrm{E}-01$ & $8,50 \mathrm{E}-01$ & 0,0 & $8,50 E+00$ & 0,0 & $8,50 \mathrm{E}-01$ & 0,0 & $8,50 \mathrm{E}-01$ & 0,0 \\
\hline $\begin{array}{l}\text { Ptrans } \\
\left(\mathrm{N} / \mathrm{m}^{2}\right)\end{array}$ & $2,94 \mathrm{E}+05$ & $7,85 E+05$ & $3,88 \mathrm{E}+05$ & & & $3,88 \mathrm{E}+05$ & 0,0 & & & & \\
\hline $\mathrm{Pt}\left(\mathrm{N} / \mathrm{m}^{2}\right)$ & $4,54 E+05$ & $6,85 E+05$ & $5,88 \mathrm{E}+05$ & & & & & $5,88 \mathrm{E}+05$ & 0,0 & $5,88 E+05$ & 0,0 \\
\hline & Dados & a rede & & & & & & & & & \\
\hline $\begin{array}{l}\text { Extensão } \\
\quad(\mathrm{m})\end{array}$ & 1873,00 & 1873,00 & 1873,00 & 1873,00 & 0,0 & 1873,00 & 0,0 & 1873,00 & 0,0 & 1873,00 & 0,0 \\
\hline $\begin{array}{l}\text { Volume } \\
\text { total }\left(\mathrm{m}^{3}\right)\end{array}$ & 227,25 & 254,57 & 235,37 & 254,54 & $-8,1$ & 229,52 & $-2,5$ & 235,37 & 0,0 & 235,37 & 0,0 \\
\hline $\begin{array}{l}\text { Volume } \\
\text { gás }\left(\mathrm{m}^{3}\right)\end{array}$ & 0,02 & 2,55 & 1,18 & 0,214 & $-81,8$ & 0,708 & $-39,8$ & 0,149 & $-87,3$ & 0,060 & $-95,0$ \\
\hline $\begin{array}{l}\text { Volume } \\
\text { líquido } \\
\left(\mathrm{m}^{3}\right)\end{array}$ & 227,23 & 252,03 & 234,19 & 252,03 & 7,6 & 228,81 & $-2,3$ & 235,22 & 0,4 & 235,31 & 0,5 \\
\hline $\begin{array}{l}\text { (\%) gás } \\
\text { adotado }\end{array}$ & 0,01 & 1 & 0,50 & 0,085 & $-83,0$ & 0,309 & $-38,3$ & 0,064 & $-87,3$ & 0,025 & $-95,0$ \\
\hline $\begin{array}{l}\text { (\%) total } \\
\text { de líquido }\end{array}$ & 99,00 & 99,99 & 99,50 & 99,92 & 0,4 & 99,69 & 0,2 & 99,97 & 0,4 & 99,97 & 0,5 \\
\hline $\begin{array}{c}\rho \mathrm{ar} \\
\left(\mathrm{kg} / \mathrm{m}^{3}\right)\end{array}$ & 1,20 & 1,25 & 1,23 & 1,23 & 0,0 & 1,23 & 0,0 & 1,23 & 0,0 & 1,23 & 0,0 \\
\hline$\underset{\left(\mathrm{kg} / \mathrm{m}^{3}\right)}{\rho \text { mistura }}$ & 926,35 & $1.049,91$ & 975,11 & 975,11 & 0,0 & 976,98 & 0,2 & 1.049 .33 & 7,6 & 979,75 & 0,5 \\
\hline
\end{tabular}

$\mathrm{R}^{\circ}=$ Resultado da otimização do Solver; $\operatorname{Var}^{\text {*⿻ }}=$ Variação em (\%); Valores arredondados

A Fig. 7 mostra a variação da celeridade em função do percentual de gás presente na mistura, que re- sulta de cada uma das formulações empregadas, e o resultado particular objetivado para a instalação. 


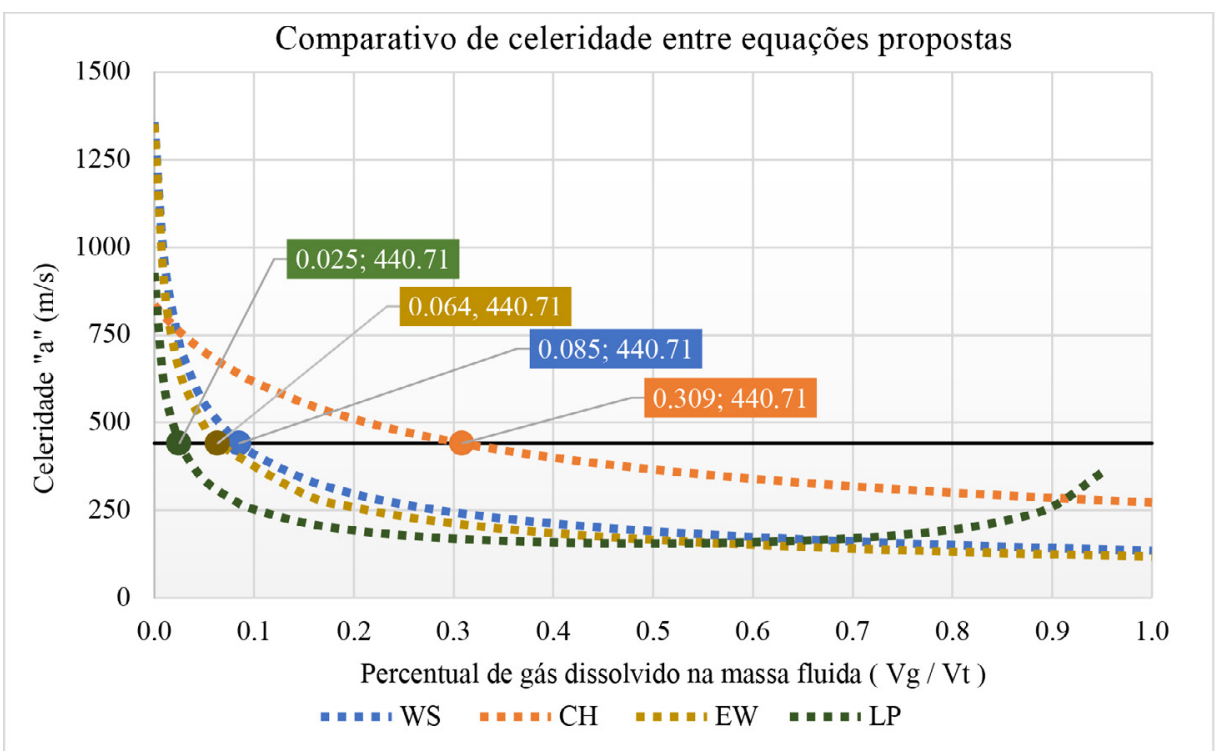

Figura 7 - Influência da variação do volume de gás dissolvido em relação à celeridade para as equações estudadas.

\section{DISCUSSÃO}

Pela análise da tabela de dados obtidos, considerando as quatro formulações analisadas, é possível observar que cada uma considera distintas formas de equacionar a celeridade para misturas líquido e gás. Apenas a equação $a_{C H}$ considerou as pressões atuantes durante o evento transitório, Ptrans na sua formulação, enquanto as equações $a_{E W}$ e $a_{L P}$ consideraram a pressão em regime permanente, $P$ t. Para que as estimativas iniciais dos valores das celeridades fossem coerentes, adotaram-se no estudo os mesmos valores das componentes e, assim, os valores iniciais foram discrepantes entre si. A maior diferença percentual negativa foi para $a_{L P}$ que foi de $-64,7 \%$. Na análise das variáveis presentes em cada equação, sem considerar o percentual de gás na mistura, observou-se que $a_{w s}$ teve três das variáveis alteradas da estimativa inicial, sendo umas delas a massa específica do líquido $\rho l$, o maior valor percentual alterado, cerca de $7,1 \%$, valor este atribuindo também para a mesma variável $\rho l$ na equação $a_{\mathrm{EW}}$, que teve apenas essa variável alterada. Já a equação $a_{C H}$ teve apenas duas variáveis modificadas em relação à estimativa inicial, a variável diâmetro da tubulação $D$ e a variável de espessura da parede $e$, ambas com $1,3 \%$ com aumen- to e decréscimo, aparentando ser a equação mais estável. Um fato que vale destacar é que três das quatro equações tiveram a adequação da variável diâmetro $D$ espessuras de parede e alteradas.

Para a análise apenas do percentual de gás, observa-se que, de forma proporcional aos valores iniciais da celeridade, as equações que tinham valores mais distantes da celeridade observada em campo foram as que tiveram maiores percentuais de gás alterados na sua solução, em ordem crescente, $a_{C H}, a_{W S}, a_{E W}$ e por fim $a_{L P}$.

Por análise gráfica é possível observar que basicamente três equações possuem comportamentos semelhantes, $a_{W S}, a_{E W}$ e $a_{L P}$ até aproximadamente $\alpha=0,5 \%$. Nota-se que $a_{C H}$ possui comportamento em partes distinto, entre $0,05 \%<\alpha<0,3 \%$ com inclinação diferenciada em relação às outras equações, possivelmente porque em sua formulação levam-se em conta as pressões finais do fenômeno transitório, PTRANS.

\section{CONCLUSÕES}

Pela verificação gráfica, conforme a Fig. 7, foi possível observar que as equações $a_{W S}, a_{E W}$ e $a_{\mathrm{LP}}$ foram 
as que mais se assemelharam ao "gráfico referência”. A equação $a_{C H}$ com cerca de $0,309 \%$ de volume de gás dissolvido ficou distante em termos de comportamento, evidenciando que sua quantidade de variáveis maior em relação às outras equações pode ser a razão de tal discrepância de comportamento. Dentre as equações que mais se assemelharam ao "gráfico referência", as que possuem trajetórias praticamente idênticas, variando o percentual de volume de gás na mistura $\left(V_{\text {gas }}\right)$, temos as equações $a_{\mathrm{wS}}$ e $a_{\mathrm{EW}}$. Assim, a primeira conclusão é de que, das equações analisadas, as que mais representam o comportamento da instalação de recalque do presente estudo são as propostas por Wylie e Streeter (1978) e Ewing (180). Sendo assim, qualquer uma das duas equações poderia ser aplicada para o estudo do fenômeno do transitório hidráulico em uma elevatória de esgotos que possuísse características semelhantes às da elevatória Jardim Jacira.

Por fim, e não por coincidência, uma vez estabelecido que as equações $a_{w S}$ e $a_{E W}$ são as que representam melhor a elevatória em estudo, o teor de gás obtido pelo processo de otimização usado representa a realidade do sistema. Assim, é possível estabelecer que o percentual de gás dissolvido na mistura de esgotos sanitários, tomando como uma média os dois resultados de tais equações, está em torno de $0,0745 \%$, valor esse que pode ser usado como parâmetro para outros estudos do fenômeno para sistemas de características semelhantes.

\section{CONTRIBUIÇÃO DOS AUTORES}

Todos os autores contribuíram de forma igualitária.

\section{REFERÊNCIAS}

ABREU, J. M.; GUARCA, R.; IZQUUIERDO, J. Transitórios y oscilaciones en sistemas hidráulicos a presión. $1^{\circ} \mathrm{Ed}$. Universidade Politécnica de Valencia, Espanha, 1995. ISBN: 84600914659788460091462

ALLIEVI, L. Teoria generale del moto perturbato dell' acqua nei tubi in pressione. Estratto dagli Atti, Associazione eletrotécnica Italiana. Roma, Itália, 1903. http://digit.biblio.polito.it/id/eprint/1119
BOULOS, P. F.; KARNEY, B.W.; WOOD, D. J.; LINGIREDDY, S. Hydraulic transient guidelines for protecting water distribution systems. Journal American Water Works Association, v. 97, n.5, p. 111124, 2005. https://doi.org/10.1002/j.1551-8833.2005.tb10892.x

BURROWS, R.; QIU, D. Q. Effect of air pockets on pipeline surge pressure. Proc. Instn Civ. Engrs Wat., Marit. \& Energy, p. 349-361, 1995.

CHAUDHRY, M. H. Applied hydraulic transients, $1^{\circ}$ Edition, Van Nostrand Reinhold, New York. 1979.

ELBASHIR, M. A. M.; AMOAH, S. O. K. Hydraulic transient in a pipeline using computer model to calculate and simulate transient. Dissertação (Mestrado em Construção e Tecnologia Ambiental). Lund University, Sweden, 2007.

EWING, D. J. F. Allowing for free air in water hammer Analysis. Proc., 3rd Int. Conf. On Pressure Surges, BHRA, Canterbury, U.K., pp. 127-146, 1980.

GHIDAOUI, M. S.; ZHAO, M.; MCLNNIS, D. A.; AXWORTHY, D. H. A Review of Water Hammer Theory and Practice. Journal Applied Mechanics Reviews, V. 58, p. 49-76, 2005. https://doi. org/10.1115/1.1828050

KÖKSOY, O.; YALCINOZ, T. Mean square error criteria to multiresponse process optimization by a new genetic algorithm. Applied Mathematics and Computation, v. 175, n. 2, p. 1657-1674, 2006. https://10.1016/j.amc.2005.09.011

LEE, T. S.; PEJOVIC, S. Air Influence on Similarity of Hydraulic Transients and Vibrations. ASME Journal of Fluids Engineering, Vol.118, No.4, December 1996, pp. 706-709. https://doi. org/10.1115/1.2835499

LUBBERS, C. L.; CLEMENS, F. Air and gas pockets in sewerage pressure mains. Delft University of Technology, Faculty of Civil Engineering and Geosciences, Section of Sanitary Engineering, Holland, 2005.

LÜDECKE, H. J.; KOTHE, B. KSB Know- how Water Hammer, Volume 1, KSB, 2006.

MALEKPOUR, A.; PAPA, F.; RADULJ, D.; KARNEY, B. Understanding hydraulic transients in sewer systems. WEAO Technical Conference, Toronto, Ontario, p. 1-15, 2015.

MOTULSKY, H. J.; RANSNAS, L. A. Fitting curves to data using nonlinear regrssion: a practical and nonmathematical review. Departament of Pharmacology, University of California, USA, 1987. https://doi.org/10.1096/fasebj.1.5.3315805

PARMAKIAN, J. Waterhammer analysis. Dover Publication, Denver, 1955.

POTHOF, I. W. M.; TUKKER, M.; KOOJI, K.; CLEMENS, F. Guidelines for capacity reducing gas pockets in wastewater mains. Technische Universiteit Delft, Holland, 2011. ISBN 0-9539140-6-2

WYLIE, E. B.; STREETER, V. L. Fluid Transients. McGraw-Hill, USA, 1978. 